\title{
Le prêtre-roi des Evhé du Sud-Togo
}

The Priest-King of the Ewe of Southern Togo

\section{Albert de Surgy}

\section{CpenEdition}

\section{Journals}

Édition électronique

URL : http://journals.openedition.org/span/888

DOl : $10.4000 /$ span. 888

ISSN : 2268-1558

\section{Éditeur}

École pratique des hautes études. Sciences humaines

\section{Édition imprimée}

Date de publication : 1 décembre 1990

Pagination : $93-120$

ISSN : 0294-7080

\section{Référence électronique}

Albert de Surgy, "Le prêtre-roi des Evhé du Sud-Togo », Systèmes de pensée en Afrique noire [En ligne], 10 | 1990, mis en ligne le 25 juin 2013, consulté le 19 avril 2019. URL : http://journals.openedition.org/ span/888 ; DOI : 10.4000/span.888 


\section{LE PRETRE-ROI DES EVHE DU SUD-TOGO}

par

\section{Albert de Surgy}

Peut-on appeler rois les chefs traditionnels éminents placés çà et là, en pays évhé', à la tête de sortes de tribus regroupant les populations de plusieurs villages? Je laisse à d'autres le soin d'en décider après examen de leur statut et de la singulière autorité qu'ils exercent. Toujours est-il qu'ils ne méritent pas d'être négligés dans l'élaboration d'une théorie de la sacralité du pouvoir.

Les Evhé se sentent en effet entourés par une multitude de puissances qui, à titre de causes efficaces ou finales, achèvent de déterminer le devenir, par lui-même très incertain, du monde de la vie sur terre. Afin d'exercer une réelle domination sur leurs semblables, il leur semble nécessaire d'entrer en rapport avec de telles puissances pour se les concilier, pour en profiter ou pour les commander. Compte tenu de cette conception du monde tout pouvoir est lié à leurs yeux aux sources du sacré et ne se légitime qu'en se donnant pour sacré.

On pourrait penser qu'un tel ancrage de l'autorité dans l'invisible est de nature à favoriser l'oppression et l'arbitraire. Cependant le sacré en question s'impose à tous les vivants. Les premiers à lui être soumis sont les chefs eux-mêmes et la condition qui leur est faite est

Par rapport à la notation évhé officielle utilisée au Togo et au Ghana, nous avons adopté là transcription suivante: $\mathcal{\nu}=0$, $v=v h, f=p h$. 
si astreignante qu'à moins de trouver satisfaction à sacrifier son existence au service de la communauté, nul n'a l'ambition d'acquérir ce statut. En cas de vacance du trône, nous n'assistons pas à une sourde lutte entre candidats pour obtenir la faveur des conseils de notables, mais éventuellement à une fuite de successeurs potentiels pour échapper au sort dont ils se jugent menacés.

L'oppression et l'arbitraire ne risquent dans ces conditions de l'emporter qu'en cas d'usurpation du sacré. Or, d'une part, celuici se situe dans le prolongement jusqu'au monde mythique des origines d'une tradition vivante collectivement sauvegardée et rénovée, d'autre part, les institutions chargées d'en authentifier les manifestations associent des représentants de tous les groupes concernés. L'usurpation en question est donc rendue impossible.

Si cérémonieuse soit-elle, une consécration destinée à mettre un chef en rapport avec des puissances invisibles, n'autorise pas à le tenir lui-même pour sacré. Toutefois, contrairement aux autres chefs ordinaires ( $f i a$ ), les grands chefs (fiagâ) traditionnels des Evhé ne se contentent pas de servir d'intermédiaires avec une source invisible d'autorité légitime. Il semble qu'ils soient eux-mêmes rendus sacrés, qu'ils incarnent une fonction divine dont ils seraient les symboles vivants efficaces.

\section{Le prêtre-roi des Bè}

Je centrerai mon propos sur le chef traditionnel des Evhé du sud du Togo qui, comme tous les autres "grands chefs" évhé, domine un certain nombre de chefs ordinaires de grand quartier ou de village ${ }^{2}$,

\footnotetext{
"Certains dominent également des "chefs de guerre" (avha-fia) qui sont les héritiers d'une Jivinité guerrière ayant pour origine les âmes errantes tuées au combat sous la direction d'un grand guerrier. Ils sont les représentants vivants d'ancêtres guerriers, excellents entraîneurs d'honmes. En matière d'autorité contraignante, ils font, çà et là, figure de chefs de quartier. On ne doit cependant pas les confondre avec les tenants d'un pouvoir qui serait acquis par la force. Il est totalement exclu qu'ils fassent usage de celle-ci pour être normés à une fonction gui demeure une lourde charge imposée à qui de droit par les anciens en fonction de signes divins.
} 
mais que son origine adja ${ }^{3}$, ainsi que la continuité de la tradition dont il se réclame, élève au-dessus de ses pairs. Il est le plus renommé du pays évhé. On venait et on vient toujours de loin lui demander protection, de sorte que son rayonnement déborde les limites du territoire qu'il contrôle.

Ce territoire est celui des autochtones de la région de Togoville et de Lomé dispersés à proximité du lac Togo et de la lagune qui prolonge celui-ci en direction de la capitale. On donne à ces autochtones le nom du village de Bè, devenu un simple quartier de Lomé, où vient résider ce "grand chef" dans la seconde partie de son règne. Ainsi parle-t-on des Bè ou de la tribu Bè ${ }^{\wedge}$.

Ce "grand chef" n'est pas toujours choisi dans le même lignages. Il suffit qu'il soit le fils d'une des principales épouses rituelles d'un de ses prédécesseurs'. Il doit toutefois n'être originaire ni de Togoville ni de Bè, de façon à ne pas être impliqué de près dans les perpétuelles luttes d'influence que poursuivent entre eux groupes familiaux et de quartier. Les autorités religieuses de ces deux localités le choisissent secrètement avec l'assistance de devins.

Une fois consacré il ne dispose d'aucun palais mais est tenu de vivre dans une portion aménagée $d^{\prime} u n$ reste de forêt primaire d'où tout signe de modernité est exclu. On ne s'y asseoit que sur des nattes. On ne doit pas y allumer de lampe-torche et a fortiori y installer l'électricité. Il ne faut y porter ni chaussures, ni bracelet-montre, ni chemise, ni pantalon. Les visiteurs ne peuvent y pénétrer que la poitrine dénudée, les reins ceints d'un petit pagne.

\footnotetext{
${ }^{3}$ Adja est le titre du roi de Tado. Par extension, il désigne courament le groupe dont les membres participent à la désignation du roi.

4 Elle comprend principalement les ressortissants de Togoville, Bè, Baguida, Dekpo, Abobo, Lebe, Dzable, Agouényivé, Mission Tové et Aflao.

"A la suite de bien d'autres, je nome ici imparfaitement "lignage" le groupe résidentiel à dominante patrilinéaire appelé aphedome ou dzotinu, lui-même subdivisé en plusieurs "familles" (phome) regroupant localement les descendants du ventre (pho) d'une même ä̈eule.

6 Les principales épouses rituelles d'un prêtre-roi lui sont fournies par ses familles paternelle et maternelle.
} 
Puisqu'il est le maître de cette forêt, on lui décerne le titre d'aveto ou d'avefia ("propriétaire" ou "chef de la forêt"). Pour ma part, je le désigne volontiers par le terme de prêtre-roi car il y remplit essentiellement les fonctions de grand-prêtre du dieu Nyigblê (les Anlo? prononcent Nyigbla). Nyigblê est le forgeron de Dieu. Il se manifeste au ciel par des étoiles filantes et des chutes d'aérolithes. A l'aide de ses outils il peut briser ce qui fait obstacle à l'arrivée des pluies et à la génération. Il libère les énergies terrestres. Le Nyigblê de Bè, jugé femelle, fait surtout fonction de dieu de la pluie et de la fécondité. Celui d'Anloga, jugé mâle, fait plutôt fonction de dieu de la contrainte sociale et de la guerre. Le même nom est par ailleurs donné à la divinité identifiée par les outils du forgeron.

Une trentaine de jeunes épouses rituelles, fiasi ("épouses du chef") parfois appelées adjamesi ("épouses de chez l'adja"), séjournent avec lui en forêt par promotions successives. Elles ne sont autorisées à porter que des ceintures de perles et, les jours de cérémonie, à se nouer également autour des reins une large bande d'étoffe dont les extrémités retombent par devant en leur dissimulant le sexe. Elles ont été mises à sa disposition par diverses familles de la tribu, habituellement en remplacement d'une de leurs grand-mères défuntes. Dans certains cas leur famille en a fait choix spontanément, dans d'autres cas elle a dû se soumettre au choix de la grand-mère en question qui, pour le faire connaître, a rendu l'intéressée malade.

Sous la direction d'une ancienne fiasi, faisant auprès d'elles fonction de "mère" et recevant le titre de fiasiwodada, elles apprennent les chants, les danses et les manières qui plaisent à Nyigblê. Elles sont progressivement aussi mises au courant de la plupart des secrets de son culte.

En aucun cas elles ne sont estimées être réellement et définitivement épousées par le prêtre-roi. Sous peine de mort prématurée, il est interdit à celui-ci d'avoir avec elles le moindre contact sexuel. Les plus âgées atteignent d'ailleurs à peine l'adolescence. Une fois rendues

"Groupe tribal évhé dont le roi, anciennement prêtre de Nyigbla lui aussi, demeure à Anloga. 
à la vie civile à l'issue de ce qui n'est en fait qu'une période d'initiation analogue à celle des adeptes de vodu appelées vodusi, elles se marient dans les mêmes conditions que n'importe qui.

A des fins pratiques cette fois, une vieille femme de sa génération est placée auprès du prêtre-roi pour prendre soin de sa santé et lui servir de maîtresse de maison (apheng). Il ne doit pas non plus avoir avec elle de rapports sexuels.

L'un de ses neveux utérins lui est adjoint comme gardien des lieux et de sa personne. Il a charge de filtrer et d'introduire auprès de lui les visiteurs. En tant que conducteur de la pirogue dans laquelle il a fallu le transporter sur le lac Togo et la lagune pour lui éviter de toucher terre, il reçoit le titre de devhuame.

Enfin un de ses jeunes frères ou le fils d'un frère de même mère que lui, appelé fionøvi, lui est adjoint pour s'occuper des problèmes de subsistance des habitants de la forêt sacrée. Il fait office de comptable et de trésorier.

\section{Une royauté purement symbolique}

Réclusion définitive, interdiction de travailler, interdiction d'avoir des rapports sexuels, en bref séparation complète d'avec le monde de la production, ne rendent pas séduisante aux yeux d'éventuels candidats la fonction de prêtre-roi. Ne sont guère disposés à l'assumer, et ne sont effectivement choisis pour l'assumer, que des hommes âgés ayant déjà été jusqu'au bout de l'expérience normale de la vie. Déjà convertis aux réalités de l'au-delà, de tels anciens ne sont plus intéressés par les affaires courantes du monde mais par celles des aïeux et des divinités. Ils ne sauraient être chefs au sens d'entraîneurs d'hommes au combat ou au travail.

En sa qualité de personnage sacré, un prêtre-roi ne peut plus avoir un contact direct avec la population. Les visiteurs qui viennent requérir ses conseils ou plutôt sa bénédiction doivent s'adresser à lui en passant par un ou souvent deux porte-parole successifs qui ont aussi à leur transmettre ses réponses. Comment un dialogue pourrait- 
il être soutenu dans de telles conditions? Qu'à cela ne tienne, son rôle majeur est d'"envoyer la parole" à destination de son dieu. Il se comporte en prêtre suprême et non en chef d'Etat.

Il faut à ce propos souligner que, du point de vue évhé, les décisions les plus importantes sont d'ordre rituel. Un homme n'est pas directement responsable de ses actes. Il est poussé à agir par les divinités, les esprits et les entités spirituelles qui l'environnent. Sa seule et grande responsabilité est de se placer sous bonne influence et de bien disposer à son égard, par une multitude d'actes de culte, les divers agents qui, depuis l'au-delà, orientent ou perturbent ses activités. De ce fait, à proportion de sa puissance et de sa notoriété, un chef est avant tout un chef religieux. Sa mission est de faire planer sur ses sujets une atmosphère bénéfique. Il est moins là pour prendre des décisions concrètes que pour faire régner la concorde. Indépendamment de lui, un pouvoir exécutif fonctionne, assuré par des conseils de notables délégués par les familles ou les quartiers. Lui-même ne fait que présider aux réunions de tels représentants du peuple en témoignant à l'occasion devant eux d'un discernement qu'il doit au contact avec l'au-delà établi lors de sa consécration et en les faisant bénéficier de l'énergie spirituelle qui émane de sa personne. Il n'est nullement nécessaire à l'expédition des affaires courantes et, à la limite, paraît même totalement étranger au gouvernement de ses sujets.

Nous constatons effectivement que la durée de règne d'un prêtreroi est en général précédée et suivie d'un interrègne plus long et que la population se passe volontiers de ses services pendant de nombreuses années sans s'en inquiéter le moins du monde.

En réalité il n'a pas le monopole du culte de Nyigblê dont sa période de règne ne constitue qu'un temps fort. Outre la forêt où il séjourne, et à laquelle il nous faut associer après sa mort la demeure sacrée de la femme, alors appelée mama avesi, qui lui fut accordée pour compagne, on compte à Togoville neuf sanctuaires ordinaires de Nyigblêt

\footnotetext{
' La répartition de ces sanctuaires dans les cinq quartiers de la ville est la suivante: au quartier Dokume un premier sanctuaire desservi par un prêtre appelé tantôt Atoku, tantôt Fi̊ggbi, un second appelé Bayi, desservi par un prêtre et une prêtresse, et un troisième appelé Aqome,
} 
pouvant être desservis par huit prêtres et trois prêtresses ${ }^{9}$. Parmi ces prêtres et prêtresses, on ne trouve cependant habituellement en fonction qu'un peu moins de la moitié d'entre eux. En effet, ils ne sont jamais immédiatement remplacés après leur mort.

Chacun de ces Nyigblê possède un nom propre et est généralement caractérisé par un lieu ou un objet particulier. Celui du prêtre-roi est symbolisé par dagbui, le python royal, animal que l'on entretient soigneusement à proximité de lui et qu'il est interdit de tuer dans toute la tribu. Un enterrement solennel, suivi de purifications, doit être réservé à ce python si on le rencontre mort chez soi, en chemin ou dans son champ. En conséquence la forêt où le prêtre-roi finit ses jours est appelée dagbuive ("forêt du python") et le quartier voisin de ceux qui ont charge d'en assurer l'entretien, dagbuiphe.

L'autorité politique et religieuse permanente reste entre les mains d'assemblées plus ou moins étendues composées de ceux et de celles qui, après l'avoir installé sur son trône (en l'occurrence une simple butte de terre recouverte d'une peau de léopard), vivent dans son orbite $^{\prime \prime}$ et dont il se retrouve littéralement prisonnier: prêtres et prêtresses en exercice, épouses rituelles de leurs prédécesseurs ou d'un précédent prêtre-roi, anciens représentant les propriétaires de chaque sanctuaire particulier, assurant l'entretien des lieux sacrés, l'organisation matérielle du culte, et participant à la désignation puis à la surveillance des prêtres.

\footnotetext{
desservi par un prêtre. Au quartier Xetiatiavi un sanctuaire appelé Ekpo desservi localement par une prêtresse, mais aussi par un prêtre résidant à Abobo, et un second appelé Aphu ("la mer"), desservi par une prêtresse. Au quartier Ayakaphe deux sanctuaires appelés Asse et Ekpe, desservis chacun par un prêtre. Au quartier Galaphe un sanctuaire desservi par un prêtre appelé tantôt Atiwoto, tantôt Âvegli. Au quartier Asiko un sanctuaire appelé Anomega, desservi par un prêtre.

${ }^{9}$ On remarquera qu'avec le prêtre-roi (puis sa compagne), cela nous donne un total de douze prêtres potentiels.

10 L'un des prêtres (Atoku ou Figgbi) est chargé d'organiser ces assemblées. Il revient à chaque groupe lignager d'y déléguer les personnes de son choix selon la nature et l'importance des questions à examiner. Rien n'empêche la réalisation entre les groupes de coalitions ou d'ententes préalables.
} 
J'appris à mes dépens l'importance de ces Anciens (les amega ou "grandes personnes humaines") sous l'influence desquels des prêtres pourtant bien disposés à mon égard refusèrent de me parler et durent revenir sur leur décision de me laisser assister à certains rites. Ayant éduqué et installé les prêtres dans leurs fonctions afin de tirer avantage de leurs pouvoirs spirituels, ils les maintiennent fermement sous leur contrôle".

On comprend que, dans ces conditions, les voyageurs français de la fin du siècle dernier appelèrent aussi volontiers républiques les petits royaumes de la côte.

Des assemblées générales, auxquelles participent des représentants de toutes les subdivisions importantes de la société, peuvent être réunies sur demande, en cas de problèmes exceptionnels, mais tiennent si j'ose dire leurs sessions ordinaires deux fois par an: en février-mars quand il convient de propitier les divinités, et en début d'automne quand il convient de les remercier de leurs faveurs. Ces dernières réunions sont l'occasion de demander publiquement à Afa (divinité des géomanciens) '" quelles sont les nouvelles de l'année (ou plutôt du semestre), de porter un jugement sur ce qui s'est passé au cours des mois précédents et de prendre plusieurs décisions dont celles jugées les plus importantes sont d'ordre rituel.

Les institutions politiques, administratives et judiciaires traditionnelles fonctionnent de la sorte sous l'impulsion de préoccupations religieuses. Mais qu'y a-t-il de si extraordinaire à cela ? Ce qui est considéré comme le plus sacré est en effet la vie harmonieuse du groupe et, par inférence, le principe qui en garantit la cohésion et la puissance. Comment différencier dès lors le culte d'un tel principe, auquel les ancêtres ont pour mission de relier leurs descendants, d'avec

\footnotetext{
- Pour échapper à l'opposition de ces Anciens, il arriva au prêtre de Nyigbla d'Anloga de venir me parler en plein milieu de la nuit sans que personne ne le sache.

1) De telles consultations se norment dufakaka, "consultations d'afa pour toute la cité". A Togoville elles ont lieu à Akuiganu, au quartier Xetiavi, lieu où l'on débat des affaires de la ville et où le prêtre-roi est présenté au public pour la première fois. Le gros de la population peut se regrouper sur la petite place située en contre-bas.
} 
la réalisation, souvent laborieuse, de la concorde entre tous et de l'adéquation des conduites aux exigences de la tradition?

\section{Un roi plus efficace mort que vivant}

On dit prudemment, ici ou là, que le prêtre-roi ne vit pas longtemps et qu'au bout de sept ans au plus tard, il est rituellement mis à mort ${ }^{13}$.

Tel est le sort qui était également réservé au roi de Notse (Nuatja) ${ }^{14}$, ville sous la domination de laquelle étaient placés tous les Evhé au début du $18 \mathrm{e}$ siècle.

Il est évidemment impossible d'enquêter directement sur le bienfondé de telles rumeurs. Cependant, en recoupant des informations recueillies séparément auprès de plusieurs prêtres ordinaires de Nyigblê et d'anciens de leur famille concernant tantôt le traitement rituel et la propre activité du prêtre-roi, tantôt le séjour en forêt de ses fiasi, je suis parvenu à établir une chronologie théorique de son règne (présentée dans le tableau ci-dessous) qui en prévoit effectivement la mort à une date déterminée.

La consécration d'un nouveau prêtre-roi et ses deux premières présentations au public, à deux jours d'intervalle, ont lieu à Togoville, dans la cour appelée akuiganu où se tiennent les réunions traitant des affaires de la ville, au cours de la période de nuit noire qui sépare la 11ème et la 12ème lune, soit sept lunes après le début de l'année traditionnelle'", à l'époque où la constellation des Pléiades, après son

\footnotetext{
13 Selon J. Bertho (1949: 42), si le prêtre-roi "n'observe pas la stricte continence conjugale, il est empoisonné. On l'empoisonne également si les récoltes sont mauvaises plusieurs années de suite, car c'est une preuve certaine qu'il ne remplit pas fidèlement son office d'intercesseur auprès des divinités et des ancêtres. Il semble même établi... qu'il ne devait pas rester en charge plus de 7 ans ( 3 ans à Porto Séguro et 4 ans à Bè). Au bout du septennat il était empoisonné".

'1 "Les nouveaux souverains, écrit Nicoué Gayibor, à compter du règne d'Asiga, ne pouvaient dorénavant régner que trois années après lesquelles ils étaient rituellement mis à mort" (Gayibor, 1982: 95)

13 Les quatre premières lunes, durant lesquelles l'année est estimée être en gestation, ne sont pas prises en considération. L'année débute donc avec la lune déclarée cinquième. Elle se poursuit durant dix lunaisons (de la cinquième à la quatorzième) suivies d'une période dite de
} 
accultation par le soleil puis par les nuages de la saison des pluies, fait sa réapparition à l'ouest après le coucher du soleil. C'est ainsi que l'intronisation du dernier prêtre-roi débuta en nouvelle lune, le 7 juillet 1967.

A l'issue de sa seconde présentation en public, ne pouvant fouler la terre sous peine de la stériliser en la brûlant ${ }^{16}$, il est transporté en pirogue de l'autre côté du lac, dans une première forêt sacrée appelée agomeve (la "forêt du rônier"), située à l'est de Porto Seguro, et y séjourne durant exactement 33 lunaisons (et non durant trois ans). C'est ainsi que le dernier prêtre-roi la quitta le 8 mars 1970.

Il est ensuite déplacé, toujours en pirogue ${ }^{17}$, jusqu'à la forêt sacrée appelée dagbuive où il doit séjourner jusqu'à sa mort. Cependant on laisse passer six lunaisons avant d'annoncer officiellement l'événement, dès le début de septembre, en envoyant l'ensemble des fiasi, accompagnées de quelques anciens, parcourir toutes les tribus voisines du littoral. Cette annonce ne s'achève qu'à la fin de la septième lunaison suivant son installation à Bè (dagbuive), c'est-à-dire 40 lunaisons après sa consécration à Togoville ${ }^{19}$, par l'envoi d'une délégation restreinte de fiasi à Notse qui est considéré comme le berceau du peuple évhé.

\footnotetext{
"mauvaises lunes" comprenant selon les cas deux ou trois lunaisons auxquelles aucun numéro d'ordre n'est attribué.

is Pour ses petits trajets terrestres, de son lieu de stationnement aux rives du lac ou de la lagune, ou inversement, il faut dérouler devant lui des nattes sur lesquelles il ne progresse qu'à une allure et avec une démarche de caméléon.

"Mais le dernier prêtre-roi fut, exceptionnellement, par conmodité, transporté secrètement à Bè en taxi au milieu de la nuit.

${ }^{19}$ Quarante est chez les Evhé un nombre complet. On comptait autrefois par paquets de quarante. Ainsi quarante cauris constituaient une unité monétaire appelée hoka. Il est la mesure d'un cycle reconduisant à une position analogue à une position de départ.
} 


\begin{tabular}{|c|c|c|c|}
\hline Date & $\begin{array}{l}\text { Nombre de lunes } \\
\text { écoulées depuis } \\
\text { sa consécration }\end{array}$ & $\begin{array}{l}\text { Evénement } \\
\text { ou } \\
\text { cérémonie }\end{array}$ & $\begin{array}{l}\text { Nombre de lunes écou- } \\
\text { lées depuis l'introduc- } \\
\text { tion en forêt de la se- } \\
\text { conde promotion de ses } \\
\text { épouses rituelles (fiasi) }\end{array}$ \\
\hline $\begin{array}{l}7 \text { juillet } \\
1967\end{array}$ & 0 & $\begin{array}{l}\text { Consécration et première présen- } \\
\text { tation du prêtre-roi à Togoville. }\end{array}$ & \\
\hline $\begin{array}{l}8 \text { mars } \\
1970\end{array}$ & 33 & $\begin{array}{l}\text { Après son initiation dans la forêt } \\
\text { Agomeve, près de Porto-Seguro, } \\
\text { le prêtre-roi, transféré à Bè, y est } \\
\text { présenté au public. }\end{array}$ & \\
\hline $\begin{array}{c}4 \text { septembre } \\
1970\end{array}$ & 39 & $\begin{array}{l}\text { Départ des fiasi pour annoncer le } \\
\text { règne du prêtre-roi dans le pays. }\end{array}$ & \\
\hline $\begin{array}{l}9 \text { octobre } \\
1970\end{array}$ & 40 & $\begin{array}{l}\text { Départ de quelques fiasi pour } \\
\text { Notse (centre de dispersion des } \\
\text { Evhé). }\end{array}$ & \\
\hline $\begin{array}{l}\text { Du } 24 \text { octobre } \\
\text { au } 3 \text { novembre } \\
1970\end{array}$ & 41 & $\begin{array}{l}\text { Levée d'interdits pour la } \\
\text { première promotion de fiasi. }\end{array}$ & \\
\hline $\begin{array}{l}29 \text { janvier } \\
1971\end{array}$ & 44 & $\begin{array}{l}\text { Dispersion de la première pro- } \\
\text { motion de fiasi et introduction en } \\
\text { forêt de nouvelles fiasi. }\end{array}$ & 0 \\
\hline $\begin{array}{l}26 \text { septembre } \\
1973\end{array}$ & 77 & Mise à mort du prêtre-roi* & 33 \\
\hline $\begin{array}{c}22 \text { janvier } 1974 \\
\text { et }\end{array}$ & 81 & $\begin{array}{l}\text { Levée d'interdits pour la seconde } \\
\text { promotion de fiasi. }\end{array}$ & 37 \\
\hline 20 janvier 1974 & 82 & $\begin{array}{l}\text { puis scarification de l'ensemble } \\
\text { des fiasi. }\end{array}$ & 38 \\
\hline $\begin{array}{l}15 \text { septembre } \\
1974\end{array}$ & 88 & $\begin{array}{l}4 \text { ans après l'annonce de son } \\
\text { règne, voyage des fiasi à Azizànu } \\
\text { (pour accompagner laâme du } \\
\text { prêtre-roi au-delà de la Volta). } \\
\text { Dispersion immédiate des } \\
\text { dernières fiasi de la forêt. }\end{array}$ & 44 \\
\hline
\end{tabular}

* Jusqu'en janvier 1971 les dates ci-dessus furent effectivement respeclées. Le prêtre-roi bénéficia d'un sursis de sept ans avant d'être mortellement brûlé dans sa case. Les cérémonies de scarification de ses épouses rituelles (fiasi) prirent fin en janvier 1981 . 
Les fiasi ayant jusqu'alors vécu auprès de lui sont rendues à leurs familles quatre lunaisons plus tard, après avoir subi une initiation étendue sur $44(=4.11)$ lunaisons ${ }^{19}$. Elles sont alors remplacées en forêt par une seconde promotion de fiasi.

C'est après avoir séjourné non pas quatre ans, mais durant 44 (= 4.11) lunaisons dans sa forêt sacrée de Bè, c'est-à-dire non pas sept ans, mais $77(=7.11)$ lunaisons après sa consécration, que le prêtreroi doit finalement mourir ${ }^{20}$.

Cependant ses funérailles ne prennent fin, avec l'accompagnement de son âme jusqu'à l'embouchure de la Volta, lieu d'embarquement pour l'au-delà, que 11 autres lunaisons plus tard, soit $88(=8.11)$ lunaisons après sa consécration. La seconde promotion de fiasi, qui se disperse à cette occasion, aura donc été soumise, elle aussi, à une initiation de 44 lunaisons.

En réalité, si la première partie du règne du dernier prêtre-roi s'est bien déroulée comme prévu, il n'a cependant pas été mis à mort au cours de la septième année de son règne mais a été maintenu en vie sept ans de plus et a subi alors une fin peu commune puisqu'il a été brûlé mortellement dans l'incendie de sa case ${ }^{21}$. Ce supplément de règne est-il dû à une répudiation moderne du régicide ou au fait que son prédécesseur fut prématurément mis à mort au bout de quatre ans seulement pour avoir eu (selon J. Bertho) un rapport sexuel avec une de ses plus grandes fiasi ? Il est bien difficile de le savoir. Toujours est-il que, de passage au Togo à l'époque où il devait être envoyé dans l'autre monde, j'ai trouvé à Togoville et à Bè une

\footnotetext{
${ }^{19}$ Le nombre 4 est jugé femelle, et le nombre 3 mâle.

20 Selon le R.P. J. Bertho il est empoisonné. Le dernier acheva de succomber à l'hôpital de brûlures consécutives à l'incendie de sa case. Enfin une personne très avertie me laissa entendre qu'il mourait de lui-même, par persuasion ou intimidation, après qu'on lui ait présenté un oeuf de perroquet qu'il lui est rigoureusement interdit de voir. Décoction, feu et vision d'un oeuf seraient-ils trois modes de suppression adaptés aux trois titres distincts qu'il reçoit ?

$>$ Selon Spieth (1911: 147), en I'honneur du Nyigbla d'Anloga, des enfants des familles environnantes furent capturés et tués. On raconte que beaucoup furent brûlés et que leurs cendres furent jetées par les chefs dans toutes les sources d'eau potable du pays. Quelquesuns des enfants capturés furent élevés dans le temple de Nyigbla pour son service.
} 
atmosphère particulièrement tendue, si méfiante à mon égard qu'il me fut impossible d'obtenir le moindre entretien sérieux avec les prêtres de Nyigblê ou toute autre personne de leur entourage.

Même si la théorie n'est pas exactement respectée, elle n'en confère pas moins une signification à la réalité qu'elle concerne. Or cette signification est capitale car elle nous présente l'existence du prêtre-roi comme une préparation à la véritable royauté qu'il n'exercera qu'une fois mort.

Etant donné que le nombre 33 est celui de tranches de 12 heures (c'est-à-dire de jours et de nuits) que compte une initiation à Afa ou à un grand vodu (Surgy, 1981: 306-402 et Verger, 1982: 35 ss.), il y a lieu d'estimer que le séjour du prêtre-roi dans sa première forêt (aggmeve) n'est qu'une préparation initiatique au règne qu'il accomplit à Bè dans sa seconde forêt (dagbuive).

Les sept lunaisons $(=6+1)$, au cours desquelles, bien qu'installé dans cet te seconde forêt, et donc initié, il n'est pas encore parfaitement déclaré tel, sont à mettre en parallèle avec la période estimée à trois mois ( six demi-lunaisons) qui sépare une initiation à Afa de la cérémonie d'ultime purification de l'initié à l'issue de laquelle il lui est seulement permis, s'il le désire, de retourner habiter chez lui.

Enfin les 37 autres lunes de son règne, réparties sur trois années luni-solaires comprenant successivement 13,12 , et à nouveau 12 lunaisons, sont à mettre en parallèle avec la période complémentaire de trois ans à l'issue de laquelle il est mis fin, par de grandes cérémonies au cours desquelles sont installés chez lui les Afamevodu nécessaires au bon exercice de son métier, à la période de formation du jeune géomancien auprès de son maître.

Cela signifie que la date à laquelle il est bon que le prêtre-roi meure est analogue à la fin d'une période de formation ayant fait suite à un cycle de simple initiation. Tout se passe comme s'il était soigneusement formé pour n'exercer qu'une fois mort le rôle qu'on attend de lui, celui d'un irremplaçable intermédiaire avec l'au-delà, élevé au rang de divinité. Si les interrègnes sont aussi longs, c'est qu'il est jugé plus efficace mort que vivant, mais ne le demeure que durant un certain 
cycle temporel à l'issue duquel son successeur doit à son tour être sacrifié pour le remplacer.

\section{L'intronisation du prêtre-roi et l'ajustement du calendrier}

L'année traditionnelle évhé (subdivisée en dix lunaisons complétées par une période dite de "mauvaises lunes") débute en principe avec la lune, appelée 5ème lune, qui suit l'apparition, à l'est, après le coucher du soleil, de la constellation des Pléiades ${ }^{\%}$, autrement dit avec la lune qui est postérieure à la courte période de visibilité de ces dernières pendant toute la nuit. Cependant, à défaut d'instruments astronomiques de précision, la détermination de cette lune reste souventapproximative ${ }^{23}$; elle dépend notamment du dégagement del'horizon et de l'opinion selon laquelle il faut ou non se baser sur l'invisibilité des Pléiades aux premiers instants de la nuit plutôt que sur leur toute nouvelle visibilité au ras de l'horizon. Il faut donc qu'une autorité incontestée se prononce. Cette autorité est celle des prêtres de Nyigblê auxquels il revient de mettre tout le monde d'accord sur le début de l'année. Une fois leur décision prise à ce sujet, ils fixent les dates des cérémonies du culte de leur dieu et c'est en se référant à elles que sont programmées celles de tous les autres cultes du pays.

La nécessité astronomique à laquelle ils ne sauraient échapper dans l'exercice de cette fonction capitale est la suivante: du fait que 19 années solaires comportent à peu près exactement $(12.19)+7=235$ lunaisons (cycle de Méton) ${ }^{\cdots a}$, la minimisation du décalage entre des années luni-solaires comportant un nombre entier de lunaisons et des années solaires (celles des saisons agricoles) est assurée en insérant sept années de 13 lunes parmi 12 autres années de 12 lunes.

\footnotetext{
22 Quand la même lune sera pleine, elle brillera à côté de la constellation des Pléiades.

23 Aux alentours de 1967 les Anlo s'estimaient en gème lune quand les Bè ne s'estimaient qu'en 7ème lurie.

$\therefore 4$ Ce n'est qu'au bout de 220 ans que le cycle de 235 lunaisons est en avance d'un jour sur le cycle de l'année tropique (ou équinoxiale).
} 
Il est remarquable qu'en novembre 1966, avant que le dernier prêtre-roi, le 15ème de la dynastie, ne soit désigné puis consacré au cours de l'année qui s'annonçait, le tout premier quartier de la lune se montrait en même temps que se levait le soir, à l'est, la constellation des Pléiades $^{2 n}$. Nous nous trouvions alors exactement à la fin d'un cycle luni-solaire de plusieurs années (de 19 ans par la force des choses) déterminé en considérant les positions respectives de la nouvelle lune et des Pléiades.

Cette observation permet de risquer l'hypothèse d'une liaison entre la nécessité ressentie par les autorités religieuses d'introniser un nouveau prêtre-roi et leurs problèmes d'ajustement du calendrier. Chaque fois que l'apparition d'une nouvelle lune ne pourrait être déclarée antérieure ou postérieure au lever héliaque des Pléiades, qui sont assimilées à la matrice du monde, le moment paraîtrait venu de consacrer un nouveau prêtre-roi dont l'existence ou le sacrifice serait de nature à redonner une impulsion au cycle du temps pour éviter qu'au lieu de se déployer il ne réintègre son origine ${ }^{26}$.

S'il en était ainsi, un intervalle de 19 ans séparerait normalement les dates d'intronisation de deux prêtres-rois. Tel n'est pas rigoureusement le cas. En particulier le 14ème prêtre-roi fut désigné beaucoup plus tôt que prévu (mais son prédécesseur avait dû être supprimé avant d'avoir achevé son temps) et nous attendons toujours que le successeur de celui qui fut intronisé en 1967 (mais auquel fut accordé un sursis de sept années) le soit à son tour ${ }^{2 \prime}$. Cependant il n'est pas exclu que des difficultés de choix d'un candidat, des rivalités entre familles,

's Date astronomique de la nouvelle lune : le 12/11/65. Date du premier quartier (marquant le début du mois lunaire traditionnel) : le 17/11/66. Date du lever héliaque des Pléiades dont I'étoile la plus brillante, Alcyone, possède une ascension droite de $3 \mathrm{~h}$. $45 \mathrm{mn}$. : le 17/11/66.

26 On observe que c'est huit ans après le début de l'année d'intronisation du prêtre-roi, c'est-à-dire deux lunaisons après la date théorique de l'accompagnement de son âme jusqu'aux rives de la Volta, que la date de la nouvelle lune s'approche à nouveau le plus (par exemple, après le $12 / 11 / 66$, le $14 / 11 / 74$ ), tout au long du cycle de 19 ans, de la date du lever héliaque des Pléiades.

$\%$ Des discussions étaient en cours à ce sujet en 1989. 
des accidents de santé, des bouleversements divers ou des réajustements faisant suite à des transgressions de la norme ne viennent perturber la règle sans altérer pour autant, sur de longues périodes, la conformité idéale qu'elle suppose aux régularités astronomiques.

Suivant notre hypothèse, sachant que le 15ème prêtre-roi fut intronisê en 1967, le 13ème aurait dû être intronisé en 1929. Or, selon le R.P. Bertho, il fut élu en 1927.

Le 8ème aurait dû être intronisé en $1967-(19.7)=1834$; or selon une tradition consignée par écrit dans la famille du chef Mlapa de Togoville, tradition confirmée ou reprise par Fio Agbanon (Agbanon 1934), ce fut sous son règne, en 1835, que l'autorisation fut donnée à des originaires d'Anecho de fonder Porto Seguro.

Le premier aurait dû être intronisé en $1967-(19.15)=1682$; or c'est effectivement aux alentours de cette date qu'il convient d'estimer l'implantation des Bè sur le littoral ${ }^{\text {sa }}$.

\section{Centralité et effacement de la personne royale}

Quelle que soit la validité de l'hypothèse avancée, devenue à présent quasiment invérifiable étant donné l'évolution des moeurs et des mentalités, il reste, me semble-t-il, que le prêtre-roi est en position de faire tourner symboliquement autour de lui la roue du temps et, par voie de conséquence, l'activité de la population.

Il existe en effet chez les Evhé un modèle d'organisation sociale qui, d'une part subdivise les groupes, majoritairement patrilinéaires, appelés aphedome (ou dzotinu), rattachant juridiquement leurs membres à une même portion de territoire, en quatre sous-groupes appelés phome, rassemblant localement les descendants de quatre ventres (pho) maternels distincts, d'autre part les réunit par trois

\footnotetext{
${ }^{28}$ Nous reportons en annexe un bref résumé de l'argumentation pouvant être soutenue en faveur de cette datation.
} 
en unités quasi-autonomes d'échanges matrimoniaux appelés hlg (ou sâme $)^{20}$.

Alors que les phome et les aphedome n'ont à leur tête que des "vieux" (ametiti) faisant fonction d'arbitres entre les vivants et d'intermédiaires avec leurs ancêtres, les hlg sont dirigés par des chefs ( fia ou fig) et se placent habituellement eux-mêmes, idéalement par groupes de douze, sous l'autorité d'un grand chef (fiaga $)^{30}$. Ce dernier n'est autre que le chef du hlg reconnu plus puissant ou plus éminent que les autres (en raison, sur le littoral, de son origine adja).

C'est ainsi que les Anlo, sujets du roi d'Anloga, se déclarent spontanément, à l'occasion de cérémonies publiques, partagés en douze $h l \varrho$ répartis en trente-six $(=3.12)$ territoires. Pour marquer symboliquement le rattachement de leurs rois (par les femmes) à toutes les sections du pays, ils ont tenu à donner aux titres qu'ils leur décernent une périodicité de douze ${ }^{31}$.

Bien que les Bè ne mettent pas en avant leur répartition en hlo, nous avons déjà fait observer qu'ils sont dirigés par un collège de prêtres et de prêtresses dont le nombre, ajouté à celui de leur grandprêtre, atteint virtuellement douze. De même que la fonction de chef est souvent héritée (en passant de l'oncle au neveu utérin) à tour de

\footnotetext{
"Le terme aphedome (ou dzotinu) est habituellement traduit par "lignage" (bien que l'endogamie y soit tolérée), le terme phome par "famille" (au sens large du terme), et hlo, (ou sâme) par "clan".

30 Aux douze hlo du pays correspondent les douze phome d'un même hlo. Alors que l'aphedome correspond sur le plan supérieur à la "maison" (aphe), dirigée par son responsable appelé apheto, le hlo, dont l'unité est assurée par les femes, correspond sur un plan supérieur à la phome fondée par une femme.

31 Voici la liste des rois anlø, résidant à Anloga : $1^{\circ} \mathrm{Sri}$, du clan Adzovia ; $2^{\circ}$ Adeladza (son neveu), du clan Bate ; $3^{\circ}$ Zanyido, du clan Adzovia ; $4^{\circ}$ Agodgmatu, du clan Bate; $5^{\circ}$ Fiayidzihe, du clan Adzovia; $6^{\circ}$ Akotsui, du clan Bate ; $7^{\circ}$ Nditsi, du clan Adzovia ; $8^{\circ}$ Aholu, du clan Bate; $9^{\circ}$ Atia, du clan Adzovia ; $10^{\circ}$ Ahiasa, du clan Bate ; $11^{\circ}$ Letsa Gbagba, du clan Adzovia ; $12^{\circ}$ Amedor Kpegla (... - 22/7/1906), du clan Bate ; $13^{\circ}$ Sri II (1907-1956), du clan Adzovia ; $14^{\circ}$ Adeladza II (1958-....), du clan Bate.
} 
rôle par les trois aphedome (ou dzotinu) constitutifs du hlo (ou sâme) ${ }^{32}$, leur chef suprême reçoit successivement les titres de Huve, Togbo et $A z \hat{a} h u$, et ainsi de suite ${ }^{3.3}$, correspondant à trois portions distinctes de la forêt sacrée où il réside. Enfin, il a de bonnes chances d'être choisi dans l'un des trois groupes lignagers d'une population étroitement apparentée, formant une sorte de hlo. En effet, toute épouse rituelle (fiasi) remplace une de ses grand-mères (de préférence maternelle); une petite-fille est de préférence mariée, pour en hériter aisément, dans la même famille que sa grand-mère; les principales épouses rituelles du prêtre-roi lui sont confiées par ses familles paternelle et naternelle; et il doit lui-même être le fils d'une ancienne épouse rituelle principale.

Parmi les douze subdivisions de l'unité politique idéale, celle à laquelle se rattache le chef suprême occupe une position centrale analogue à la partie invaginée (comme retranchée elle aussi au coeur de la forêt ${ }^{34}$ ) d'une période de $12.3=36$ années lunaires dont ne se fait voir que le cycle de 33 années lunaires (de douze lunaisons), à l'issue duquel le début de l'année lunaire a presque rétrogradé d'un tour complet sur le cycle de l'année solaire (il ne s'en écarte plus que de 6,214 jours), et où il semble avoir besoin de reprendre vigueur.

Il suffirait que des spécialistes du calendrier aient élevé au rang de paradigme cette quasi-équivalence de 33 années lunaires à 32 années solaires, ou années réelles des cultivateurs, pour avoir été conduits à estimer que la perpétuation de tout cycle fondé sur la distinction de douze catégories ne pouvait être assurée que par une impulsion exercée à son origine depuis l'une de ses catégories spécia-

\footnotetext{
32 Cf. Verdon, 1983: 65, 95 et 260 , note 25 . Le successeur du premier roi anlo (du hlq Adzovia) fut un neveu utérin gui appartenait à un autre hlo (du nom de Bate). Depuis lors le roi est choisi à tour de rôle dans le hlq Adzovia puis Bate.

33 Traitant de la royauté à Notse, Nicoué Gayibor (op. cit., p. 96) nous fait part d'informations analogues selon lesquelles "la royauté devint rotative entre les trois grands quartiers: Avizuha (Alinu), Agblaladome et Ekli où les rois étaient choisis à tour de rôle. Il en sera ainsi, dit la tradition, jusqu'à la veille de la conquête coloniale."

$3 n$ En effet le prêtre-roi n'habite pas exactement une forêt sacrée mais une clairière circulaire aménagée en pleine forêt (aujourd'hui dans ce qui reste préservé de l'ancienne forêt).
} 
lement escamotée à cet effet par retrait au centre. En cette catégorie singulière viendraient se confondre et se souder les premières et dernières catégories des cycles successifs. Il en résulterait la conception d'un cycle dynamique de onze unités et une restructuration des 33 années lunaires totalisant (3.11).12 = (3.12) .11 = 396 lunaisons, en 36 cycles de onze lunaisons. Or ce sont bien de tels cycles de onze (se reporter au tableau de la page 10) qui servent au décompte de la durée du règne du prêtre-roi comme de l'initiation de ses jeunes épouses rituelles.

Position centrale du clan royal lui permettant de fournir symboliquement une impulsion au cycle calendaire toutes les 33 années lunaires

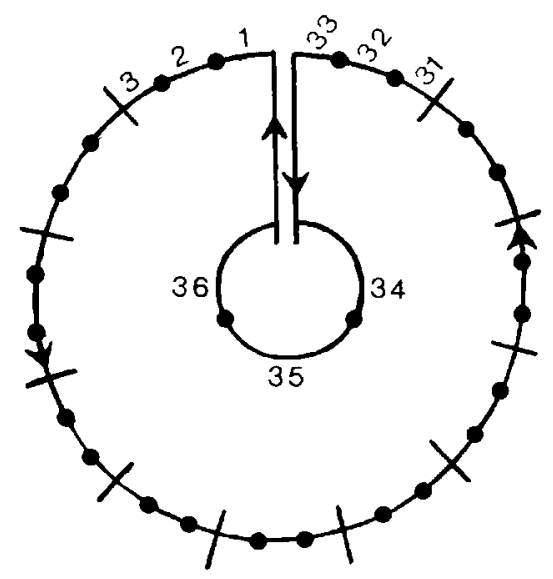

A supposer qu'une telle conception ait prévalu, le renouvellement du compte du temps qui intervient tous les 19 ans (235 lunaisons) ${ }^{35}$, se traduisant par une sorte de remise à zéro des compteurs, impliquerait que les impulsions secrètes conférées au cycle lunaire toutes les onze lunaisons soient suspendues à compter de la vingt et unième,

\footnotetext{
35 En réalité la prenière approximation satisfaisante du cycle luni-solaire, celui où un nombre entier de cycles de douze lunaisons coïncide avec un nombre entier d'années solaires, est une période de douze cycles de Méton valant $19.12=228$ années solaires ou 235.12 lunaisons, c'est-à-dire $228+7=235$ années lunaires. Cette période se subaivise en 7 cycles de 33 années lunaires complétés par un reste de 4 années lunaires, ou en 256 cycles de 11 luaisons (autant de cycles de 11 lunaisons que de figures géomantiques utilisées en divination par Afa) complétés par un reste de 4 lunaisons (en effet, $235.12=2820$ lunaisons $=256.11+4$ lunaisons $=2816$ +4 lunaisons).
} 
c'est-à-dire au bout de $21.11=7.33=231$ lunaisons, pour ne reprendre qu'avec un retard de quatre lunaisons. A cet effet l'impulseur en exercice paraît devoir être empêché d'agir et remplacé par un autre. C'est effectivement, dans l'intervalle entre les 7ème et 8ème lunes, date habituelle des cérémonies de Nyigblê à Togoville, préparées par des réunions de responsables du culte, que semble normalement désigné un nouveau prêtre à consacrer et présenter au public quatre lunes plus tard ${ }^{36}$.

Ce personnage sacré représente un principe divin (1'artisan forgeron mythique Nyigblê) qui harmonise les activités des deux grands distributeurs des forces vitales dans le monde que représentent la lune et le soleil, qui garantit la pérennité des cycles temporels et du renouvellement des générations les unes par les autres, assure l'articulation et l'assemblage des éléments de la mécanique cosmique ou sociale et, de ce fait, se situe en dehors d'elle, y demeurant totalement irrepérable ${ }^{3 x}$.

A un tel principe une partie de l'énergie normalement engagée dans la production mérite d'être sacrifiée, mais ce sacrifice ne se distingue pas de la construction, suivie de la suppression, du symbole vivant de son destinataire. C'est par une participation de toutes les familles à l'élaboration puis à la vénération de ce symbole que la société locale

\footnotetext{
36 Cette dernière durée de 4 lunes (nombre associé à la terre et aux ténèbres procréatrices) articule un règne au suivant comme elle articule une année à la suivante et un grand cycle de 228 ans (256 fois 11 lunaisons, plus 4 lunaisons) au suivant.

37 La fonction démiurgique assurée chez les Mwaba-Gurma du nord-Togo par le Dieu-Soleil yêdu, puissance d'unification en acte dans le monde, semble assurée chez les Adja-Evhé, par le forgeron divin qui libère à l'origine des temps les puissances génératrices (ouverture du sexe féminin ou brisure de la montagne empêchant les flots de venir au monde) puis en orchestre le jeu. Ce forgeron représente la Force suprême qui a pouvoir de tout contenir en germe aussi bien que de tout ramener à l'unité par conciliation et assemblage. Toutes les divinités particulières (trog et vodu) lui sont subordonnées. On notera toutefois que lui est étroitement apparenté l'aspect de la Divinité mise à la disposition de chaque home pour l'aider à accomplir au mieux sa destinée et lui tenant lieu de génie individuel (appelé $\underline{\mathrm{Se}}$ ). Tout prêtre de Nyigblê, a fortiori le prêtreroi, est obligatoirement initié à Afa (divinité des géomanciens), c'est-à-dire mis en relation avec son Se. Il y aurait à élucider, par l'analyse des objets, des gestes et des textes rituels, cette relation privilégiée du roi avec le génie (que les Mwaba-Gurma connaissent aussi sous le nom de cabl ou de yêdu personnel). Elle est évoquée par Gilbert Rouget (Rouget, 1989: 192).
} 
régénère périodiquement sa propre substance et bénéficie en retour d'une vigueur qui n'a pas de prix. Elle se dote ainsi d'un point de mire collectif qui réalise d'autant mieux autour de lui l'union de tous ses membres qu'il demeure non-agissant ou est déjà passé dans l'audelà.

Déjà fortement marquée de son vivant par un retrait quasimonacal dans une portion de forêt inculte, son désengagement des affaires du monde est encore accentué après son décès, compte tenu de la nature de celui-ci, par sa transformation en "mauvais mort" de la catégorie des ezâmeku qui, enterrés de nuit sous le couvert des arbres au lieu de l'être de jour comme les autres en pleine savane, n'exercent ni une activité d'ancêtre, ni celle de fantôme errant perturbateur (Surgy, 1988: 124-125) ${ }^{3 \mathrm{~s}}$. L'exceptionnelle puissance qui lui est attribuée est en fait proportionnelle à la sorte d'annulation de lui-même à laquelle il doit consentir. C'est en effet en vertu de son abstention de toute activité engagée qu'il lui est possible de s'identifier à la grande Cause agissante du monde, retranchée de ses effets, et de se poser ainsi en libre Agent par rapport à ce qu'il advient non seulement sur terre mais aussi au pays des ancêtres et dans l'étendue intermédiaire entre les deux.

Du fait qu'il n'exerce aucun commandement, qu'il est la chose de son peuple, soumis à lui jusqu'à en devenir victime, je comparerais volontiers l'institution de son règne à l'organisation des matsuri japonais, ces imposantes festivités en l'honneur des divinités (kami) protectrices d'un certain terroir, dont la fonction sociale fut à ce point reconnue que le gouvernement du pays y reçut le nom de matsurigoto (en étymologie phonétique: "les choses des matsuri"). Comme chacun sait, la divinité vivante nationale, celle de l'ensemble du terroir, n'était

\footnotetext{
38 Son âme paraît embarquée (à l'embouchure de la Volta) pour l'autre monde, sans jamais pourtant $Y$ arriver. C'est alors qu'il mérite au plus haut point sa qualification de "roi de l'eau", ne se déplaçant que dans une pirogue. Constatant l'association du chef gourmantché (Burkina Faso) avec la brousse, Michel Cartry (1987: 213-220) a de son côté attiré notre attention sur l'état symboliquement mort avant de naitre du chef vivant et sa conservation, une fois mort, en position non ancestralisable, dans un espace de parfaite stérilité. Cette remarquable analyse converge avec la nôtre.
} 
autre au Japon que l'empereur. Or à l'époque classique les empereurs étaient démissionnés à peine sortis de l'adolescence. C'est en se dévouant alors à la personne d'un enfant, et ce fut ultérieurement en se dévouant à un empereur dépossédé de ses pouvoirs par des chefs militaires puis par une constitution démocratique, que le Japon construisit et préserva son unité.

Si nous avons le plus grand mal à admettre l'étrange pouvoir d'un souverain-objet demeurant à la merci de ceux qui l'ont installé sur son trône dans l'espoir d'en tirer avantage, si nous ne concevons aisément qu'un pouvoir dominateur, imposé d'en haut ou abandonné par nousmêmes entre les mains de mandataires, serait-ce que nous aurions perdu le sens de la véritable souveraineté populaire plongeant ses racines dans la volonté non pas répressive mais expressive de faire la fête? Toute fête digne de ce nom est en effet reconstitutive de symboles propres à capter l'attention, réunir, structurer et animer, à l'inverse de ce que produit un encadrement autoritaire.

A. de Surgy

URA 221 - (EPHE-CNRS) 


\section{Annexe relative à la date de déploiement des Evhé sur le littoral}

Quand, en 1680, en raison de leurs conflits persistants avec les Akwamu, les Gê quittèrent en grand nombre la région d'Accra, sous la direction du prince Ashangmo, pour aller chercher refuge à Glidji (Bosman, 75, 5ème lettre), ils firent alliance, au passage, avec les Anlo de Keta pour repousser des guerriers dahoméens qui, selon les uns, tourmentaient les Bè et, selon les autres, étaient leurs alliés (cf. Reindorf, p. 36, Agbanon, 1934:2, et Cornevin 1969:17). A cette date les Bè occupaient done déjà les lieux.

Cependant les Gê ne faisaient alors que rejoindre deux des leurs: Foli Bebe et Foli Hemadzo, déjà établis depuis $1663^{39}$, auxquels aucune tradition orale ne prête de tractations avec des Keta ou des Bè.

On nous dit par ailleurs que, parmi les groupes qui se dispersèrent depuis $\mathrm{Notse}^{40}$, à l'occasion de l'éclatement du royaume dirigé au $17 \mathrm{e}$ siècle par Agokoli, les Bè arrivèrent parmi les derniers sur la côte. Or il paraît impossible que les Anlo y soient arrivés avant 1650 .

Les Anlo furent en effet conduits à Keta par un meneur d'hommes appelé Wenya qui, à son départ de Notse, appartenait déjà à la génération des grand-pères". Ce Wenya se retira à Anloga sur ses vieux jours quand il fut rejoint par son neveu Kponoe Sri (fils de sa petite soeur Asogoe donnée en mariage au roi Asimedi de Tado) dont le trône dut être récupéré à Notse et qui devint le premier roi des Anlo. Or c'est toujours à Keta que W. Bosman (Voyage de Guinée, 5ème lettre) assure avoir rencontré en 1698 le chef des Keta, tout en précisant, au moment où il rédige son texte ( 4 à 6 ans plus tard), que ce village a cessé d'être sa résidence. Wenya ne put donc mourir à Anloga qu'au cours du $18 \mathrm{e}$ siècle.

"La première défaite des Gê de la région d'Accra contre les Akwamu date de 1660. Elle fut suivie du suicide (volontaire ou forcé ?) de leur roi Okaï koï.

nille située au nord de Lomé, à la latitude de Tado.

1 L'une des causes de l'exode de Notse fut une querelle, différemment expliquée par les auteurs, mettant en cause le fils du fils de sa petite soeur (séparée de lui par deux garçons). Cf. J. Bertho, op.cit.: 30 ; Muller, Geschichte der Ewe Mission, p. 106, cité par Debrunner 1965: 8 ) et D.K. Fiawoo, 1959, chap. I, "Traditions of origin". 


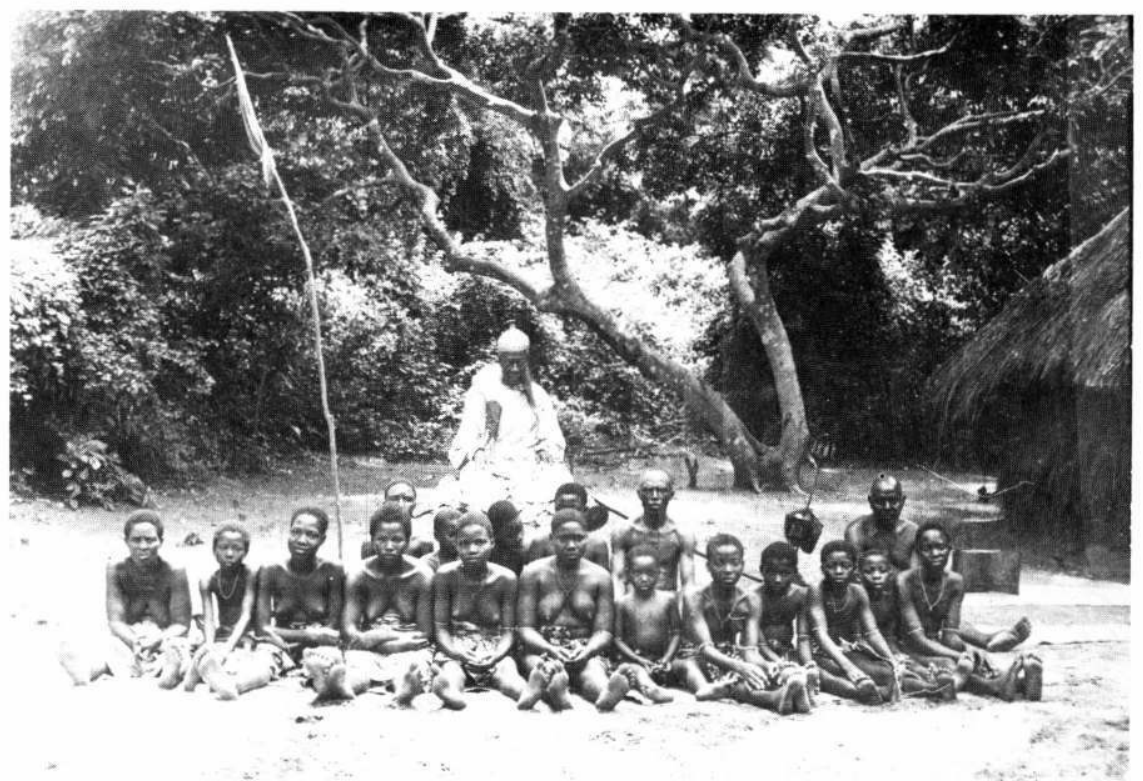

Le prêtre-roi dans sa forêt sacrée d'Agomeve, avec des fiasi, la fiasiwodada, le prêtre Atoku et le devhuame. (Cl. Surgy).

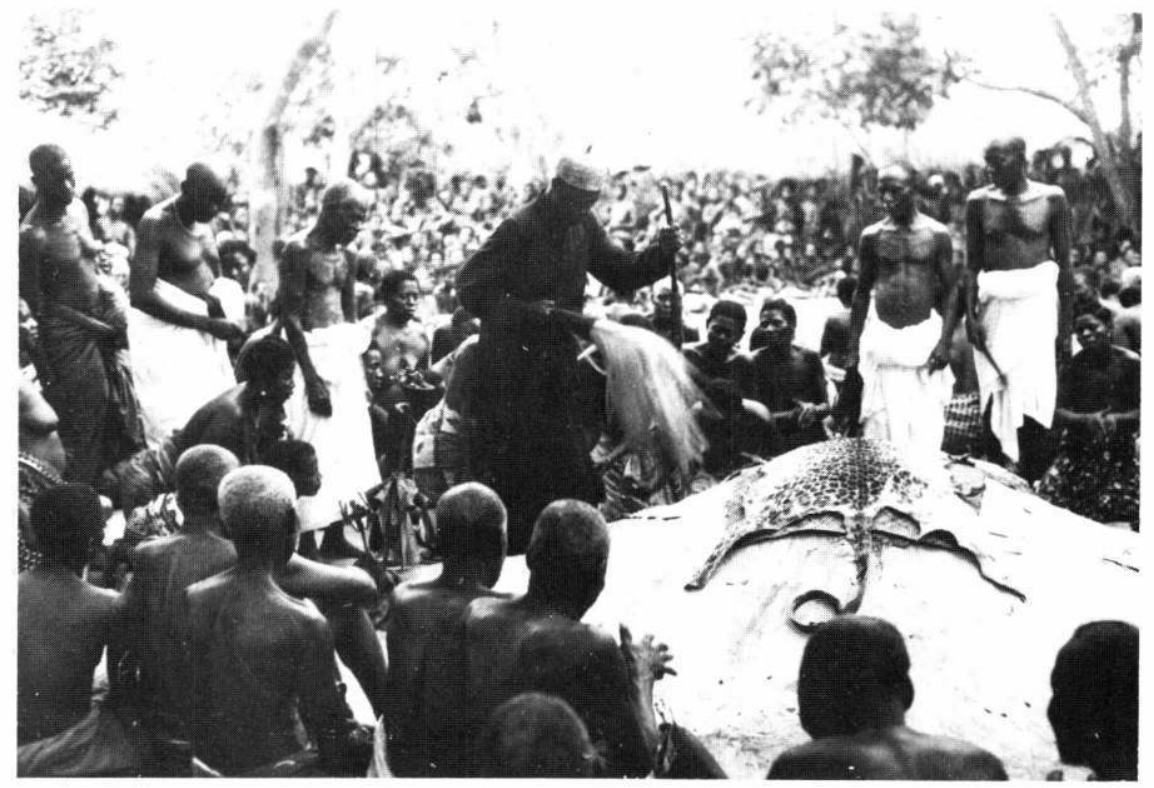

Le prêtre-roi montant trôner sur sa butte aga. (Cl. Surgy). 


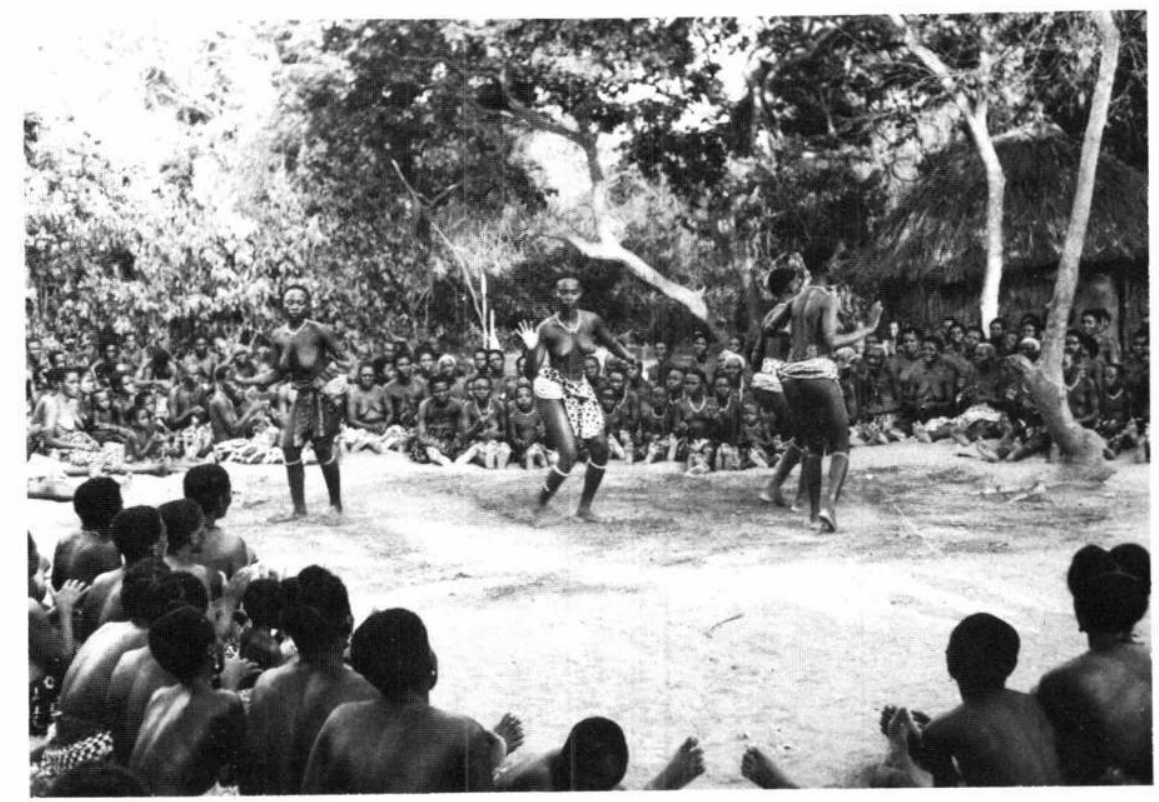

(Cl. Surgy).

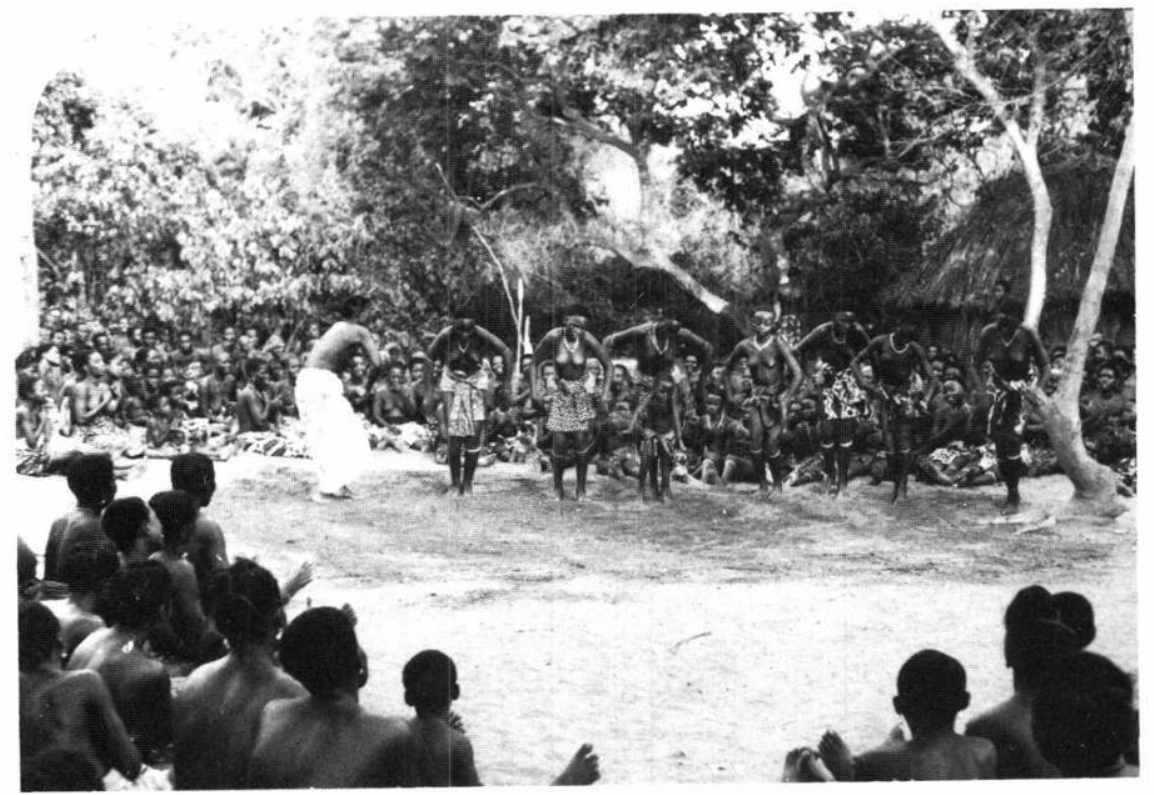

Cérémonie dans la forêt agomeve, précédant le transfert à Bè du prêtre-roi. (Cl. Surgy). 

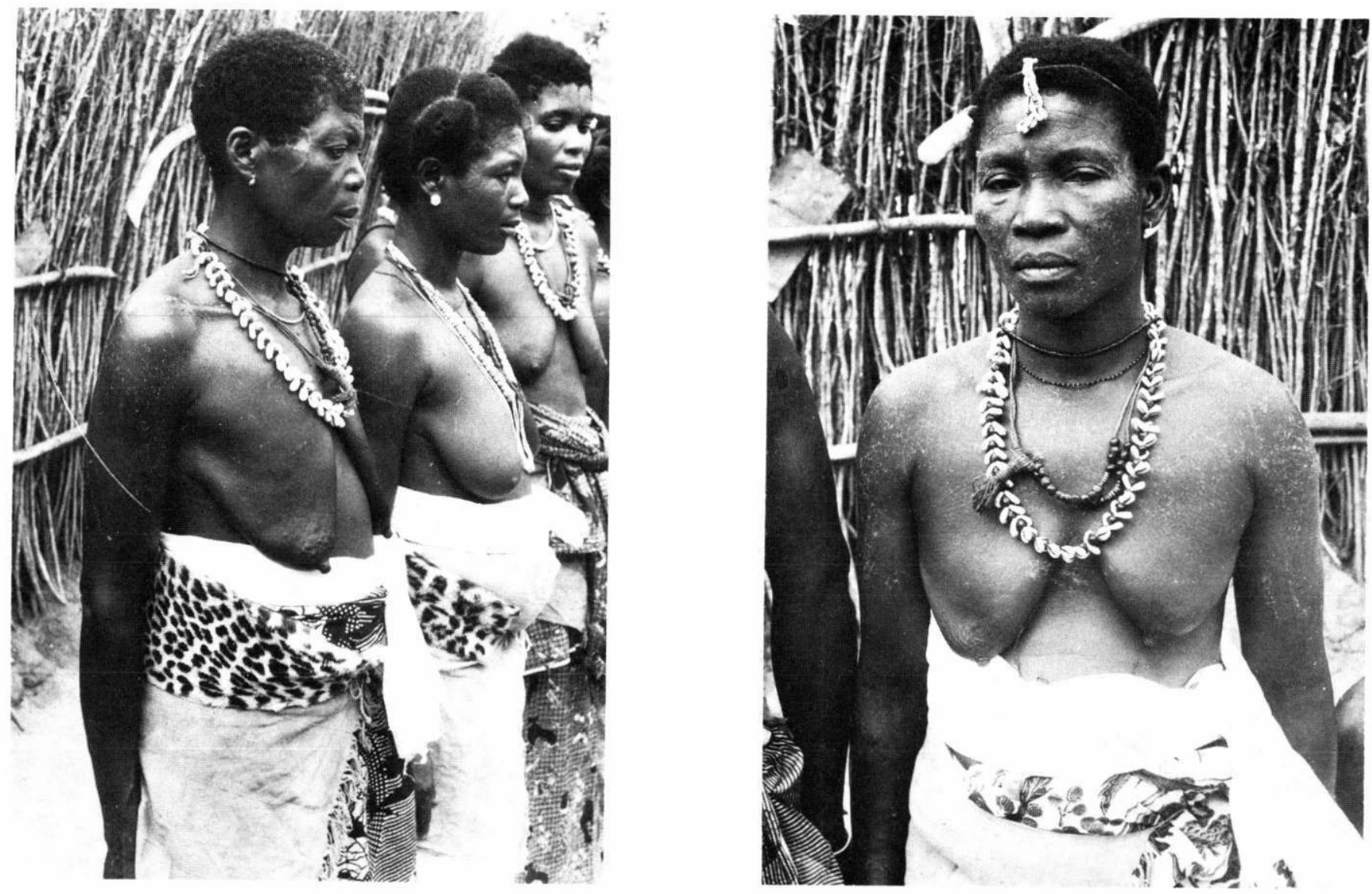

Fiasi du précédent prêtre-roi. (Cl. Surgy). 


\section{Ouvrages mentionnés}

Agbanon, Fio

1934 Mémoire sur l'histoire du Petit Popo et du peuple Gê, Lomé, texte ronéoté, $135 \mathrm{p}$.

Bertho, Rev. P. J.

1949 Etude sur la parenté des Yoruba, des Adja, des Ouatchi et des Ewe, Lomé, Imprimerie de l'Ecole Professionnelle (presque intégralement reproduit dans Africa XIX, 1949, 121132 , sous le titre "La parenté des Yorouba aux peuples du Dahomey et du Togo")

Bosman, W.

1705 Voyage de Guinée, Utrecht, Souten (1ère éd. 1702 en hollandais )

Cartry, M.

1987 "Le suaire du chef", in Sous le masque de l'animal, Textes réunis par M. Cartry, Paris, PUF, 131-231.

Cornevin, R.

1969 Histoire du Togo, Paris, Berger-Levrault, (1ère éd. 1959), $556 \mathrm{p}$.

Debrunner, H.W.

1965 A church between colonial powers, Londres, Lutterworth Press.

Fiawoo, D.K.

1959 The Influence of Contemporary Social Changes in the MagicoReligious Concepts and Organisation of the Southern Ewe Speaking Peoples of Ghana, thèse de philosophie non publiée, Edimbourg.

Gayibor, N.L.

1982 "Migrations et organisation politique des Adja-Ewe au cours de la l'ère précoloniale", Annales de l'Université du Bénin, (série Lettres), 9.

Reindorf, Rev. C.C.

s.d. "The history of the Gold Coast and Ashanti, from about 1500 to 1860", Basel Mission Book Depot, (1ère éd. 1889), 349p.

Rouget, G.

1989 "Le roi, le babouin, l'indigo", in Singularités. Textes pour Eric de Dampierre, Paris, Plon, 179-196. 
Spieth, J.

1911 Die Religion der Eweer in Süd Togo, Leipzig/Göttingen, Van der Hoeck und Ruprecht, 316 p.

Surgy, A. de,

1981 La géomancie et le culte d'Afa chez les Evhé du littoral, Paris, Presses Orientalistes de France, $444 \mathrm{p}$.

1988 Le système religieux des Evhé, Paris, L'Harmattan, 334 p.

Verdon, $M$.

1983 The Abutia Ewe of West Africa, Berlin/Amsterdam/New York, Mouton, $311 \mathrm{p}$.

Verger, $P$.

1982 Orisha, Paris, Métaillé, 293 p. 